\title{
DIFFERENCE EQUATIONS AND MULTIPOINT BOUNDARY VALUE PROBLEMS
}

\author{
P. W. ELOE
}

Abstract. Let $I=\{a, a+1, \ldots, b\}$ be finite, let $n \geqslant 1$, and let $I^{j}=\{a, a+$ $1, \ldots, b+j\}, j=1, \ldots, n$. Let $B$ be the set of mappings from $I^{n}$ into the reals and define the linear difference operator $P$ by

$$
\begin{aligned}
P u(m)= & \sum_{j=0}^{n} \alpha_{j}(m) u(m+j), \\
& \text { where } m \in I, \alpha_{n}(m) \equiv 1, \text { and } \alpha_{0}(m) \neq 0 \text { on } I .
\end{aligned}
$$

Existence of solutions theorems and iteration schemes that approximate solutions are given for boundary value problems of the form $P u(m)=f\left(m, u, E u, \ldots, E^{n-1} u\right)$, with boundary conditions $T u(m)=r$, where $P$ is defined by $(1), E^{j} u(m)=$ $u(m+j), j=0,1, \ldots, n-1, f: I \times \mathbf{R}^{n} \rightarrow \mathbf{R}$ is continuous, and $T: B \rightarrow \mathbf{R}^{n}$ is a continuous linear operator. The results are based on solutions of difference inequalities and sign properties of associated Green's functions.

1. Introduction. Let $I=\{a, a+1, a+2, \ldots, b\}$ be finite, let $n \geq 1$ and let $I^{j}=$ $\{a, a+1, \ldots, b+j\}, j=1, \ldots, n$. Define the $n$th order linear difference operator $P$ by

$$
P u(m)=\sum_{j=0}^{n} \alpha_{j}(m) u(m+j),
$$

where the independent variable $m$ ranges over $I, \alpha_{n}(m) \equiv 1, \alpha_{0}(m) \neq 0$ on $I$, and the coefficients $\alpha_{j}(m), j=0, \ldots, n$, are defined on $I$. Define the Banach space $B$ to be the set of real valued maps on $I^{n}$ with norm

$$
\|u\|=\max _{m \in I^{n}}|u(m)| \text {. }
$$

Define shift operators $E^{j}$ by the relations

$$
E^{j} u(m)=u(m+j), \quad j=0,1, \ldots, n-1, m \in I .
$$

Let $T: B \rightarrow \mathbf{R}^{n}$ be a continuous linear operator.

We shall consider the boundary value problem (BVP)

$$
P u(m)=f\left(m, u, E u, \ldots, E^{n-1} u\right) \equiv f[m, u],
$$

with boundary conditions given by

$$
T u(m)=r,
$$

where $f: I \times \mathbf{R}^{n} \rightarrow \mathbf{R}$ is continuous and $r \in \mathbf{R}^{n}$.

In [5], P. Hartman defined disconjugacy of the linear difference equation

$$
P u(m)=0,
$$

Received by the editors December 2, 1981. Presented at 88th Annual Meeting of the American Mathematical Society, in Cincinnati, January 11-17, 1982. 
where $P$ is defined by (1.1), in the following manner. For a sequence of real numbers $u: u(a), u(a+1), \ldots, u(a+b+n), m=a$ is a generalized zero for $u$ if $u(a)=0$, and $m$ $(>a)$ is a generalized zero for $u$ if $u(m)=0$ or there is an integer $k, 1 \leq k \leq m-a$, such that $(-1)^{k} u(m-k) u(m)>0$ and, if $k>1, u(m-k+1)=\cdots=u(m-1)=$ 0 . The difference equation (1.5) is disconjugate on $I^{n}$ if no solution $u \neq 0$ has $n$ generalized zeros on $I^{n}$.

Hartman [5] obtained many results that have analogues in the theory of disconjugacy of $n$th order linear differential equations. Of special interest to us, Hartman showed that the Green's function for a class of BVPs may satisfy known sign properties, as in the following theorem.

THEOREM H. Let $P$ be given by (1.1) and assume that (1.5) is disconjugate on $I^{n}$. Let $m_{1}, m_{2}, \ldots, m_{n} \in I^{n}$ such that $a=m_{1}<m_{2}<\cdots<m_{n}=b+n$. Then there exists a unique Green's function $G(m, s)$ defined on $I^{n} \times I$ such that for any function $f=f(m), m \in I$, the unique solution of the BVP

$$
P u(m)=f(m), \quad u\left(m_{i}\right)=0, \quad i=1, \ldots, n,
$$

is given by $u(m)=\sum_{s=a}^{b} G(m, s) f(s), m \in I^{n}$. Moreover, $G(m, s)$ satisfies the inequality

$$
(-1)^{n+\sigma(m)} G(m, s) \geq 0, \quad m \in I^{n}, s \in I,
$$

where $\sigma(m)=\operatorname{card}\left\{j: m_{j}<m, 1 \leq j \leq n\right\}$.

Motivated by the inequality (1.6), we define the following condition.

CONDITION S. There exists a Green's function $G(m, s)$ for the BVP $P u(m)=0$, $T u(m)=0$, and the sign of $G(m, s)$ is independent of $s$.

This paper provides results concerning existence of solutions of various classes of BVPs for which Condition S holds. In $\$ 2$ we present preliminary results from the theory of linear difference equations and an application of the Schauder fixed point theorem. In $\S 3$ we establish theorems for the existence of solutions of the BVP (1.3)-(1.4) based on Condition $S$ and solutions of difference inequalities; in addition, we provide piecewise monotone iteration schemes to approximate solutions of the BVP (1.3)-(1.4). The results of this section are analogous to some of the results of V. Seda [6] and P. W. Eloe and L. J. Grimm [1] for ordinary differential equations and Grimm and K. Schmitt [4] and Eloe and Grimm [2] for functional-differential equations.

2. Preliminary results. We shall state some results from the theory of linear difference equations; we refer the reader to the book by T. Fort [3]. Let $P$ be given by (1.1) and let $T: B \rightarrow \mathbf{R}^{n}$ be a continuous linear operator. The boundary conditions given by (1.4) are more general than those defined in Chapter IX in [3], but the following propositions remain valid if $T$ is a continuous, linear operator.

PROPOSITION 2.1. If $u \equiv 0$ is the only solution of the $B V P P u(m)=0, T u(m)=$ 0 , then for any $r \in \mathbf{R}^{n}$, there exists a unique solution of the $B V P P u(m)=0$, $T u(m)=r$.

PROPOSITION 2.2. If $u \equiv 0$ is the only solution of the $B V P P u(m)=0, T u(m)=$ 0 , there exists a unique function $G(m, s)$, called the Green's function, defined on $I^{n} \times I$ which has the following characterization: For each $s \in I, v(m)=G(m, s)$ is 
the unique solution of the $B V P P u(m)=\delta_{m s}, T u(m)=0$, where $\delta_{m s}=1$ if $m=s$ and $\delta_{m s}=0$ if $m \neq s$. Furthermore, for any function $f=f(m)$ defined on $I$, the unique solution of the BVP

$$
P u(m)=f(m), \quad T u(m)=0,
$$

is given by

$$
u(m)=\sum_{s=a}^{b} G(m, s) f(s) .
$$

From the above propositions, it follows that if $u \equiv 0$ is the only solution of $P u(m)=0, T u(m)=0$, then for any $f=f(m)$ on $I$ and $r \in \mathbf{R}^{n}$, the unique solution of the BVP

$$
\begin{aligned}
& P u(m)=f(m), \\
& T u(m)=r,
\end{aligned}
$$

is given by

$$
u(m)=l_{r}(m)+\sum_{s=a}^{b} G(m, s) f(s),
$$

where $l_{r}(m)$ is the unique solution of $P u(m)=0, T u(m)=r$.

Let $f: I \times \mathbf{R}^{n} \rightarrow \mathbf{R}$ be continuous and define the summation operator $K: B \rightarrow B$ by

$$
K u(m)=l_{r}(m)+\sum_{s=a}^{b} G(m, s) f[s, u],
$$

where $f[m, u]$ is defined as in (1.3). Note that $u$ is a solution of the BVP (1.3)-(1.4) if, and only if, $u$ is a fixed point of the operator $K$ defined by (2.4). We have the following fundamental result.

THEOREM 2.3. Suppose $u \equiv 0$ is the only solution of the homogenous BVP $P u(m)=0, T u(m)=0$ and suppose that $f: I \times \mathbf{R}^{n} \rightarrow \mathbf{R}$ is continuous and bounded. Then for any $r \in \mathbf{R}^{n}$, the BVP (1.3)-(1.4) has a solution.

Proof. Let $Q=\sup \left\{\left|f\left(m, u_{1}, \ldots, u_{n}\right)\right|:\left(m, u_{1}, \ldots, u_{n}\right) \in I \times \mathbf{R}^{n}\right\}$. Let $l_{r}(m)$ be the unique solution of $P u(m)=0, T u(m)=r$, and set $L=\left\|l_{r}\right\|$. Let $G(m, s)$ be the Green's function for $P u(m)=0, T u(m)=0$, and set $G=\max _{m \in I^{n}} \sum_{s=a}^{b}|G(m, s)|$. Let $D=\{u \in B:\|u\| \leq L+Q G\}$ and note that the continuous operator $K$, defined by (2.4), maps the closed convex set $D$ into itself and that $\overline{K(D)}$ is compact. By the Schauder fixed point theorem, the operator $K$ has a fixed point and so, the BVP (1.3)-(1.4) has a solution.

3. Existence of solutions. Let $P$ be defined by (1.1), let $T: B \rightarrow \mathbf{R}^{n}$ be a continuous linear operator and let $r \in \mathbf{R}^{n}$. Consider the BVP

$$
P u(m)=f\left(m, u, E u, \ldots, E^{n-1} u\right) \equiv f[m, u]
$$

with boundary conditions given by

$$
T u(m)=r,
$$


where the shift operators $E^{j}, j=0, \ldots, n-1$, are defined by (1.2), and $f: I \times \mathbf{R}^{n} \rightarrow$ $\mathbf{R}$ satisfies the Lipschitz condition

$$
\left|f\left(m, u_{1}, \ldots, u_{n}\right)-f\left(m, \hat{u}_{1}, \ldots, \hat{u}_{n}\right)\right| \leq Q \sum_{i=1}^{n}\left|u_{i}-\hat{u}_{i}\right|,
$$

for all $\left(m, u_{1}, \ldots, u_{n}\right),\left(m, \hat{u}_{1}, \ldots, \hat{u}_{n}\right)$ in $I \times \mathbf{R}^{n}$, for some $Q>0$. Note that if $f$ satisfies (3.3), then $f$ is continuous on $I \times \mathbf{R}^{n}$.

Assume that Condition S holds for the BVP $P u(m)=0, T u(m)=0$, and let $\left\{I_{1}, I_{2}\right\}$ be a partition of $I^{n}$ such that

$$
G(m, s) \leq 0 \text { for }(m, s) \in I_{1} \times I, \quad G(m, s) \geq 0 \text { for }(m, s) \in I_{2} \times I .
$$

Further, assume that there exist functions $v_{1}$ and $w_{1}$ in the Banach space $B$ satisfying

$$
T v_{1}(m)=r=T w_{1}(m)
$$

and such that for $m \in I$,

$$
P v_{1}(m)-f\left[m, v_{1}\right]+A_{1}(m) \leq 0 \leq P w_{1}(m)-f\left[m, w_{1}\right]-A_{1}(m),
$$

where

$$
A_{1}(m)=Q \sum_{i=0}^{n-1}\left|E^{i}\left(v_{1}(m)-w_{1}(m)\right)\right|
$$

THEOREM 3.1. Assume that Condition S holds for the $B V P P u(m)=0$, $T u(m)=0$, and suppose that $f: I \times \mathbf{R}^{n} \rightarrow \mathbf{R}$ satisfies (3.3). Assume there exist functions $v_{1}$ and $w_{1}$ satisfying (3.5) and (3.6). Then there exists a solution $u$ of the BVP (3.1)-(3.2) such that

$$
\begin{array}{ll}
v_{1}(m) \geq u(m) \geq w_{1}(m), & m \in I_{1}, \\
v_{1}(m) \leq u(m) \leq w_{1}(m), & m \in I_{2} .
\end{array}
$$

Proof. We shall first show that

$$
v_{1}(m) \geq w_{1}(m), \quad m \in I_{1}, \quad \text { and } \quad v_{1}(m) \leq w_{1}(m), \quad m \in I_{2} .
$$

By (3.5), $v_{1}-w_{1}$ satisfies $T u(m)=0$ and so,

$$
\left(v_{1}-w_{1}\right)(m)=\sum_{s=a}^{b} G(m, s)\left[P\left(v_{1}-w_{1}\right)(s)\right] .
$$

It follows from (3.6) and (3.7) that $P\left(v_{1}-w_{1}\right)(m) \leq 0$ for $m \in I$; the sign of $G(m, s)$ is given by (3.4) and the inequalities (3.9) follow.

For each $u \in B$, define $\overline{E^{j} u}, j=0, \ldots, n-1$, as follows: For $m \in I$,

$$
\overline{E^{j} u}(m)=\left\{\begin{array}{ll}
E^{j} v_{1}(m), & \text { if } E^{j} u(m)>E^{j} v_{1}(m) \\
E^{j} u(m), & \text { if } E^{j} w_{1}(m) \leq E^{j} u(m) \leq E^{j} v_{1}(m) \\
E^{j} w_{1}(m), & \text { if } E^{j} u(m)<E^{j} w_{1}(m) \\
E^{j} v_{1}(m), & \text { if } E^{j} u(m)<E^{j} v_{1}(m) \\
E^{j} u(m), & \text { if } E^{j} w_{1}(m) \geq E^{j} u(m) \geq E^{j} v_{1}(m) \\
E^{j} w_{1}(m), & \text { if } E^{j} u(m)>E^{j} w_{1}(m)
\end{array}\right\} \quad m+j \in I_{1},
$$


For $m \in I$, define $\bar{F}[m, u] \equiv f\left(m, \bar{u}, \overline{E u}, \ldots, \overline{E^{n-1} u}\right)$. The function $\bar{F}$ is continuous and bounded on $I \times \mathbf{R}^{n}$ and so, by Theorem 2.3, the BVP $P u(m)=\bar{F}[m, u]$, $T u(m)=r$, has a solution $u$.

We now show that the solution $u$ of the truncated problem $P u(m)=\bar{F}[m, u]$, $T u(m)=r$, satisfies (3.8) and hence that $u$ is a solution of $(3.1)-(3.2) .\left(v_{1}-u\right)(m)$ satisfies $T\left(v_{1}-u\right)(m)=0$; thus,

$$
\left(v_{1}-u\right)(m)=\sum_{s=a}^{b} G(m, s)\left(P\left(v_{1}-u\right)(s)\right),
$$

for $m \in I^{n}$. For $m \in I$,

$$
P\left(v_{1}-u\right)(m) \leq f\left[m, v_{1}\right]-\bar{F}[m, u]-A_{1}(m) \leq 0
$$

since $f$ satisfies (3.3). The sign of $G(m, s)$ is given by (3.4) and it follows that $v_{1}(m) \geq u(m), m \in I_{1}$, and $v_{1}(m) \leq u(m), m \in I_{2}$. Similarly, $u(m) \geq w_{1}(m), m \in$ $I_{1}$, and $u(m) \leq w_{1}(m), m \in I_{2}$. Thus, $u$ satisfies (3.8) and the proof is complete.

The functions $v_{1}$ and $w_{1}$ satisfying (3.5) and (3.6) provide a priori bounds for solutions of the BVP (3.1)-(3.2). These bounds can be improved iteratively in the following manner. Let $l_{r}(m)$ be the unique solution of the BVP $P u(m)=0$, $T u(m)=r$. Define inductively the sequences $\left\{v_{j}(m)\right\}$ and $\left\{w_{j}(m)\right\}$ for $m \in I^{n}$ by

$$
\left\{\begin{array}{l}
v_{j+1}(m)=l_{r}(m)+\sum_{s=a}^{b} G(m, s)\left(f\left[s, v_{m}\right]-A_{j}(s)\right), \\
w_{j+1}(m)=l_{r}(m)+\sum_{s=a}^{b} G(m, s)\left(f\left[s, w_{m}\right]+A_{j}(s)\right),
\end{array}\right.
$$

for $j \geq 1$, where $v_{1}$ and $w_{1}$ satisfy (3.5) and (3.6) and

$$
A_{j}(m)=Q \sum_{i=0}^{n-1}\left|E^{i}\left(v_{j}(m)-w_{j}(m)\right)\right|,
$$

for $m \in I$. The proof of the following corollary can be obtained directly from the proof of Theorem 1 [1] and is omitted here.

COROLlary 3.2. Assume all the hypotheses of Theorem 3.1 and define the sequences $\left\{v_{j}(m)\right\}$ and $\left\{w_{j}(m)\right\}$ by (3.10). If $u$ is any solution of the $B V P(3.1)-(3.2)$ satisfying (3.8), then for each $j \geq 1$,

$$
\begin{array}{ll}
v_{j}(m) \geq v_{j+1}(m) \geq u(m) \geq w_{j+1}(m) \geq w_{j}(m), & m \in I_{1}, \\
v_{j}(m) \leq v_{j+1}(m) \leq u(m) \leq w_{j+1}(m) \leq w_{j}(m), & m \in I_{2} .
\end{array}
$$

REMARKS. (i) Let $G=\max _{m \in I^{n}} \sum_{s=a}^{b}|G(m, s)|$ and let $Q$ be as in (3.3). If $Q G n<1$, then $K$ defined by (2.4) is a contraction map and an application of the Contraction Mapping Principle provides existence and uniqueness of a solution of the BVP (3.1)-(3.2).

(ii) Due to the generality of the boundary conditions (3.2), we require that $v_{1}$ and $w_{1}$ satisfy (3.5). As with boundary value problems for differential equations [6], this condition can be weakened. Let $l_{v_{1}}(m)$ be the unique solution of the BVP $P u(m)=0, T u(m)=T v_{1}(m)$, and let $l_{w_{1}}(m)$ be the unique solution of the BVP 
$P u(m)=0, T u(m)=T w_{1}(m)$. Suppose

$$
\begin{array}{ll}
l_{v_{1}}(m) \geq l_{r}(m) \geq l_{w_{1}}(m), & m \in I_{1}, \\
l_{v_{1}}(m) \leq l_{r}(m) \leq l_{w_{1}}(m), & m \in I_{2},
\end{array}
$$

where $l_{r}(m)$ is the unique solution of the BVP $P u(m)=0, T u(m)=r$. Then the hypothesis that $v_{1}$ and $w_{1}$ satisfy (3.11) can replace the hypothesis that $v_{1}$ and $w_{1}$ satisfy (3.5) in Theorem 3.1. Corollary 3.2 remains valid.

(iii) If $f$ has certain monotonicity properties, then solutions of BVPs may be found as limits of approximating sequences. For example, consider the BVP

$$
\begin{aligned}
& P u(m)=f(m, u), \\
& T u(m)=r,
\end{aligned}
$$

where $P$ and $T$ are as in Theorem 3.1 and Condition $\mathrm{S}$ holds. Suppose that $f: I \times$ $\mathbf{R} \rightarrow \mathbf{R}$ is continuous and satisfies the monotonicity condition, for $u_{1}, u_{2} \in \mathbf{R}, u_{1} \geq$ $u_{2}$,

$$
f\left(m, u_{1}\right) \leq f\left(m, u_{2}\right), \quad m \in I_{1}, \quad \text { and } f\left(m, u_{1}\right) \geq f\left(m, u_{2}\right), \quad m \in I_{2} .
$$

Let $l_{r}(m)$ be the unique solution of the BVP $P u(m)=0, T u(m)=r$, and define the summation operator $K: B \rightarrow B$ by

$$
K u(m)=l_{r}(m)+\sum_{s=a}^{b} G(m, s) f(s, u(s)) .
$$

Suppose there exist $v_{1}$ and $w_{1}$ in $B$ satisfying (3.5) such that

$$
v_{1}(m) \geq w_{1}(m), \quad m \in I_{1}, \quad \text { and } \quad v_{1}(m) \leq w_{1}(m), \quad m \in I_{2},
$$

and for $m \in I$,

$$
P v_{1}(m)-f\left(m, v_{1}(m)\right) \leq 0 \leq P w_{1}(m)-f\left(m, w_{1}(m)\right) .
$$

Define inductively the sequences $\left\{v_{j}(m)\right\}$ and $\left\{w_{j}(m)\right\}$ by

$$
v_{j+1}(m)=K v_{j}(m) \text { and } w_{j+1}(m)=K w_{j}(m),
$$

for $j \geq 1$, where $K$ is defined by (3.15). Then the sequences $\left\{v_{j}(m)\right\}$ and $\left\{w_{j}(m)\right\}$ converge monotonically on $I_{1}$ and $I_{2}$ to solutions of the BVP (3.12)-(3.13). We summarize the above discussion in the following theorem.

THEOREM 3.3. Let $P$ and $T$ be as in Theorem 3.1, let Condition $\mathrm{S}$ hold, and let $f: I \times \mathbf{R} \rightarrow \mathbf{R}$ be continuous and satisfy (3.14). Assume there exist functions $v_{1}$ and $w_{1}$ in $B$ satisfying (3.5), (3.16) and (3.17) and define the sequences $\left\{v_{j}(m)\right\}$ and $\left\{w_{j}(m)\right\}$ by (3.18). Then the sequences, $\left\{v_{j}(m)\right\}$ and $\left\{w_{j}(m)\right\}$ converge in $B$ to $v(m)$ and $w(m)$, respectively, where $v(m)$ and $w(m)$ are solutions of the $B V P(3.12)-(3.13)$ and such that for each $j \geq 1$,

$$
\begin{array}{ll}
v_{j}(m) \geq v_{j+1}(m) \geq v(m) \geq w(m) \geq w_{j+1}(m) \geq w_{j}(m), & m \in I_{1}, \\
v_{j}(m) \leq v_{j+1}(m) \leq v(m) \leq w(m) \leq w_{j+1}(m) \leq w_{j}(m), & m \in I_{2} .
\end{array}
$$


(iv) Define $\Delta u(m)=u(m+1)-u(m)$ and $\Delta^{i+1} u(m)=\Delta\left(\Delta^{i} u(m)\right), i=1,2, \ldots, n-$ 1. For various classes of boundary value problems the sign of $\Delta^{i} G(m, s)$ is independent of $s$, for some $i, 1 \leq i \leq n-1$, where $\Delta G(m, s)=G(m+1, s)-G(m, s)$, $\Delta^{i+1} G(m, s)=\Delta\left(\Delta^{i} G(m, s)\right), i=1, \ldots, n-1$. For example, it can be shown that the Green's function for the BVP $\Delta^{2} u(m)=0, u(a)=\Delta u(b+1)=0$, satisfies

$$
\begin{aligned}
G(m, s) \leq 0 & \text { for }(m, s) \in I^{2} \times I, \\
\Delta G(m, s) \leq 0 & \text { for }(m, s) \in I^{1} \times I .
\end{aligned}
$$

Theorem 3.1 can be extended in a natural way to boundary value problems of the form $\Delta^{2} u(m)=f(m, u, \Delta u)$, with boundary conditions $u(a)=r_{1}, \Delta u(b+1)=r_{2}$, $r_{1}, r_{2} \in \mathbf{R}$.

(v) We can consider the difference equation (3.1) to be a functional-difference equation with advanced argument. For certain classes of boundary value problems, Theorem 3.1 can be extended to functional-difference equations with retarded and advanced argument. Such extensions are analogues of results of Grimm and Schmitt [4] and Eloe and Grimm [2] on functional-differential equations.

\section{REFERENCES}

1. P. W. Eloe and L. J. Grimm, Monotone iteration and Green's functions for boundary value problems, Proc. Amer. Math. Soc. 78 (1980), 533-538.

2. $\ldots$ Multipoint boundary value problems for functional-differential equations, Rocky Mountain J. Math. (to appear).

3. T. Fort, Finite differences, Oxford Univ. Press, Oxford, 1948.

4. L. J. Grimm and K. Schmitt, Boundary value problems for differential equations with deviating arguments, Aequationes Math. 3 (1969), 321-322; 4 (1970), 176-190.

5. P. Hartman, Difference equations: disconjugacy, principle solutions, Green's functions, complete monotonicity, Trans. Amer. Math. Soc. 246 (1978), 1-30.

6. V. Seda, Two remarks on boundary value problems for ordinary differential equations, J. Differential Equations 26 (1977), 278-290.

Department of MAThematics, UNIVERsity OF DAYTON, DAYTON, OHIO 45469 